\title{
OPERATOR RADII AND UNITARY OPERATORS
}

\author{
Tsuyoshi ANDO AND CHI-KwONG LI
}

Abstract. Let $\rho \geqslant 1$ and $w_{\rho}(A)$ be the operator radius of a linear operator $A$. Suppose $m$ is a positive integer. It is shown that for a given invertible linear operator $A$ acting on a Hilbert space, one has $w_{\rho}\left(A^{-m}\right) \geqslant w_{\rho}(A)^{-m}$. The equality holds if and only if $A$ is a multiple of a unitary operator.

Mathematics subject classification (2010): 47A20, 47A12, 15A60.

Keywords and phrases: Unitary $\rho$-dilation, operator radius, numerical radius, unitary operator.

\section{REFERENCES}

[1] T. Ando, Structure of operators with numerical radius one, Acta Sci. Math. (Szeged), 34 (1973), $11-15$.

[2] M. D. Chol AND C. K. LI, Numerical ranges of the powers of an operator, to appear in J. Math. Anal. Appl., preprint available at http://people.wm.edu/ cklixx/power.pdf.

[3] E. DurSzT, Factorization of operators in $\mathscr{C}_{\rho}$ class, Acta Sci. Math. (Szeged), 37 (1975), 195-200.

[4] J. A. R. Holbrook, On the power bounded operators of Sz.-Nagy and Foiaş, Acta Sci. Math. (Szeged), 29 (1968), 299-310.

[5] K. Okubo And T. Ando, Constants related to operators of class $\mathscr{C}_{\rho}$, Manuscripta Math., 16 (1975), 385-394.

[6] T. Sano And A. UchiYama, Numerical radius and unitarity, to appear in Acta Sci. Math. (Szeged).

[7] J. G. Stampfli, Minimal range theorems for operators with thin spectra, Pac. J. Math., 23 (1967), 601-612.

[8] B. SZ.-NagY, On uniformly bounded linear transformations in Hilbert space, Acta Sci. Math. (Szeged), 11 (1947), 152-157.

[9] B. SZ.-NAGY AND C. FOIAŞ, On certain classes of power bounded operators in Hilbert space, Acta Sci. Math. (Szeged), 27 (1966), 17-25. 